\title{
Dielectric composites with a high and temperature-independent dielectric constant
}

\author{
Xiaobing SHAN, Lin ZHANG, Xiqiao YANG, Z.-Y. CHENG ${ }^{*}$ \\ Materials Research and Education Center, Auburn University, Auburn, AL 36849, USA \\ Received: October 5, 2012; Revised: November 30, 2012; Accepted: December 1, 2012 \\ OThe Author(s) 2012. This article is published with open access at Springerlink.com
}

\begin{abstract}
Dielectric composites made using P(VDF-CTFE) 88/12 mol\% as polymer matrix and both micro-sized and nano-sized $\mathrm{CaCu}_{3} \mathrm{Ti}_{4} \mathrm{O}_{12}$ (CCTO) particles as filler are developed. These composites exhibit high dielectric constant with a small dielectric loss. More importantly, it is found that the dielectric constant of these composites is almost independent of temperature from $25{ }^{\circ} \mathrm{C}$ to $125{ }^{\circ} \mathrm{C}$. Comparing the composites made using micro-sized CCTO particles, the composites made using nano-sized CCTO particles exhibit a smaller dielectric loss. The dielectric properties of these composites indicate that the nano-sized CCTO particles have a smaller dielectric constant than the micro-sized CCTO particles.
\end{abstract}

Key words: dielectric constant; composite; $\mathrm{CCTO}$; $\mathrm{P}(\mathrm{VDF}-\mathrm{CTFE})$; temperature dependence

\section{Introduction}

Polymer-based dielectric composites are highly desirable for applications ranging from electronic packaging, embedded capacitors, to energy storage, since these composites are highly flexible with a low process temperature and they exhibit a relatively high dielectric constant, low dielectric loss, high dielectric strength $[1,2]$. A great deal of effort has been dedicated to the development of these composites. The dielectric property of a composite is strongly dependent on the connectivity of its constituents. Due to the simple preparation process and desirable flexibility, 0-3 composites are the main category of polymer based composites studied [1]. In a polymer-based 0-3

* Corresponding author.

E-mail: chengzh@eng.auburn.edu composites, polymer serves as the matrix, and the particles of other materials are embedded into the polymer matrix as filler. The filler can be either a dielectric or conductor [1,2]. The high dielectric constant obtained in the 0-3 composites using conducting filler is caused by the percolation phenomenon. The dielectric properties of a 0-3 composite using a dielectric as filler are dependent on the dielectric properties of both the matrix and filler [1]. To achieve a high dielectric constant in polymer based 0-3 composites using dielectric filler, it is necessary to have: (1) dielectric with high dielectric constant as filler and (2) polymer with high dielectric constant as matrix.

Most of dielectrics used as fillers in the development of polymer based 0-3 composites are inorganic polar materials, especially ferroelectrics [3-9], such as $\mathrm{BaTiO}_{3}$ (BT), (Ba,Sr) $\mathrm{TiO}_{3}$ (BST), $\mathrm{Pb}\left(\mathrm{Mg}_{2 / 3} \mathrm{Nb}_{1 / 3}\right) \mathrm{O}_{3}$ (PMN), $\mathrm{P}\left(\mathrm{Mg}_{1 / 3} \mathrm{Nb}_{2 / 3}\right) \mathrm{O}_{3}-\mathrm{PbTiO}_{3}$ (PMN-PT) and $\mathrm{Pb}(\mathrm{Zr}$, 
$\mathrm{Ti} \mathrm{O}_{3}$ (PZT), since these dielectrics exhibit a dielectric constant as high as $10^{3}$ to $10^{4}$ [10]. The dielectric constant can reach 100 in these composites [4,7]. However, the polar dielectrics exhibit a strong electromechanical effect, which is undesirable for dielectric applications. Additionally, the dielectric properties of the polar materials exhibit a strong temperature dependence [10], which is undesirable for many dielectric applications. Regarding polymer matrix, it is well known that most of polymers exhibit a low dielectric constant $(<5)[1,11]$. To have a polymer with a high dielectric constant, most attention has been given to PVDF-based polymers, such as PVDF homopolymer and P(VDF-TrFE) copolymers, which exhibit a dielectric constant more than of 10 at room temperature [7,11-15]. Again, it is necessary to mention the dielectric constant of these polymers exhibits a strong temperature dependence due to its ferroelectric nature. For example, the dielectric constant of P(VDF-TrFE) can reach up to 80 at the ferroelectric-to-paraelectric phase transition temperature [15], which is more than 7 times of that at room temperature. The ferroelectric-to-paraelectric phase transition temperature in $\mathrm{P}(\mathrm{VDF}-\mathrm{TrFE})$ ranges from $60{ }^{\circ} \mathrm{C}$ to $150{ }^{\circ} \mathrm{C}$ depending on the composition [16]. Therefore, the dielectric properties of a composite made of polar polymer matrix and/or polar filler are strongly dependent on the temperature.

The discovery of $\mathrm{CaCu}_{3} \mathrm{Ti}_{4} \mathrm{O}_{12}$ (CCTO) and other similar materials opens a new avenue for the development of 0-3 composites, since these materials exhibit an ultrahigh dielectric constant $\left(10^{4}-10^{5}\right)$, and their dielectric constant is almost independent of the temperature over a broad temperature range that covers the room temperature [17-19]. Additionally, these materials are not piezoelectric. CСТO has been used as filler material for the development of polymer based 0-3 composites, and CCTO-based composites exhibit a high dielectric constant [20-24]. In these composites, ferroelectric-like polymers, such as PVDF and $\mathrm{P}(\mathrm{VDF}-\mathrm{TrFE})$, have been used as polymer matrix. Therefore, the dielectric constant of these composites exhibits a strong temperature dependence. For example, for composites made of CCTO particle with $\mathrm{P}(\mathrm{VDF}-\mathrm{TrFE}) \quad 55 / 45 \mathrm{~mol} \%$, the dielectric constant at the ferroelectric-to-paraelectric phase transition temperature $\left(\sim 70{ }^{\circ} \mathrm{C}\right)$ of $\mathrm{P}(\mathrm{VDF}-\mathrm{TrFE})$ is about $2-3$ times of that at room temperature for the composite with 50 vol\% CCTO [21]. It should be mentioned that the physics behind the high dielectric constant observed in CCTO is interesting. The dielectric behavior of CCTO-based composites provides a unique way to study the origin of high dielectric constant obtained in CCTO.

In researching electroactive polymer, it is found that $\mathrm{P}$ (VDF-CTFE) copolymers have following features [25-27]: (1) similar with other PVDF-based polymer, $\mathrm{P}(\mathrm{VDF}-\mathrm{CTFE})$ copolymer at room temperature exhibit a dielectric constant about 10; (2) P(VDF-CTFE) copolymer can withstand a very high electric field. Both of these make the P(VDF-CTFE) copolymer a great candidate for energy storage. A great deal of efforts has been given to the development of energy-storage composites using $\mathrm{P}$ (VDF-CTFE) copolymer as matrix [27-30]. All this research focuses on energy storage density and dielectric properties of these composites at room temperature. It should be noticed that P(VDF-CTFE) copolymer does not show phase transition, so its dielectric constant is only weakly dependent on the temperature. This and the above two features make P(VDF-CTFE) copolymer an ideal polymer to be used as polymer matrix for the development of polymer-based 0-3 composites for dielectric applications.

In this work, 0-3 composites using CCTO particles as filler and P(VDF-CTFE) 88/12 mol\% copolymer as polymer matrix are developed. The dielectric properties and their dependences on the composition, temperature, and frequency are reported here. It is found that these CCTO-P(VDF-CTFE) composites exhibit a relatively high dielectric constant with a low dielectric loss and, more importantly, the dielectric constant of these composites is almost independent of the temperature over temperatures ranging from $25^{\circ} \mathrm{C}$ to $125{ }^{\circ} \mathrm{C}$. Both micro- and nano-sized CCTO particles were prepared using the same CCTO ceramics.

In the experiments, СCTO ceramics were prepared by using a traditional powder processing method as described in References [20,21]. CCTO particles/ powders in size about $10 \mu \mathrm{m}$ (i.e., microsized particles) were prepared from CCTO ceramics using a ball miller, while the CCTO particles in size less than $1 \mu \mathrm{m}$ (i.e., nanosized particles), mostly ranging from $100 \mathrm{~nm}$ to $600 \mathrm{~nm}$, were prepared from CCTO ceramics using a Fritsch P4TM high-energy ball milling system. P(VDF-CTFE) 88/12 mol\% copolymer was first dissolved in dimethyl formamide (DMF) using magnetic stir bar for $8 \mathrm{~h}$ at room temperature. The 
CCTO particles were then added to the polymer solution and stirred for another $8 \mathrm{~h}$ and sonicated for about $20 \mathrm{~min}$. The final CCTO/P (VDF-CTFE) suspension/solution was cast on a glass plate at $70{ }^{\circ} \mathrm{C}$ for $8 \mathrm{~h}$. The composites with $0,10,20,30,40$, and $50 \mathrm{vol} \%$ of CCTO were prepared. The as-cast film was annealed at $125{ }^{\circ} \mathrm{C}$ for $8 \mathrm{~h}$. The thickness of as-cast film is around $50-80 \mu \mathrm{m}$. To improve the uniformity of the composite, a stack of as-cast composite film with 4 layers was placed in a hot-press machine at $150{ }^{\circ} \mathrm{C}$. The stack was pressed with 10 tons force for 10 seconds. The scanning electron microscope (SEM JSM-7000F, JEOL) observation confirmed that the hot-press samples have a much better uniformity than the as-cast samples, which is consistent with the results obtained from CCTO-P(VDF-TrFE) composites [20,21]. The hot-press samples with sputtered gold electrode on both sides were used for the dielectric characterization. The morphology of the composites was examined using SEM. The impedance of the samples at frequencies ranging from $100 \mathrm{~Hz}$ to $1 \mathrm{MHz}$ over a temperature range from $25{ }^{\circ} \mathrm{C}$ to $125{ }^{\circ} \mathrm{C}$ was measured using an impedance analyzer (Agilent 4294A). The dielectric constant was calculated from the impedance using parallel plate mode.

The frequency dependences of the dielectric constant and dielectric loss for the composites at room temperature are shown in Fig. 1(a), where the composites with different CCTO contents $(0,10,20$, $30,40,50 \mathrm{vol} \%$ ) are presented and the content of CCTO is identified in the figure. Clearly, the dielectric constant of the composites increases with increasing CCTO content. The data shown in Fig. 1 indicates that CCTO-P(VDF-CTFE) composites exhibit a high dielectric constant with a low loss. For example, the composite with 50 vol\% CCTO exhibits a dielectric constant more than 150 with a dielectric loss about $13 \%$ at $1 \mathrm{kHz}$. This dielectric constant is about 18 times of that of pure $\mathrm{P}$ (VDF-CTFE) copolymer.

As shown in Fig. 1(b), the dielectric loss at high frequency $(>100 \mathrm{kHz})$ increases with increasing frequency, which is dominated by the dielectric relaxation process in the polymer matrix. The dielectric loss at low frequency $(<10 \mathrm{kHz})$ is more complicated. For the composites with low CCTO content (e.g., 0, 10, $20 \mathrm{vol} \%$ ) (named as Regime-I for later on discussion), the dielectric loss at low frequency is almost independent of the CCTO content. However, for the
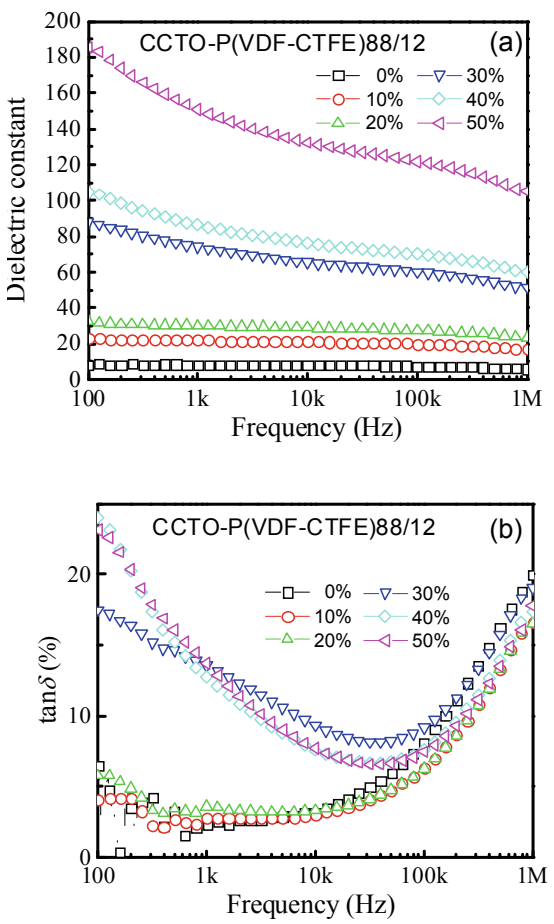

Fig. 1 Dielectric constant (a) and dielectric loss (b) versus frequency for CCTO/P(VDF-CTFE) composites at room temperature. The content of CCTO $(0,10,20,30,40,50 \mathrm{vol} \%)$ in the composites is indicated in the figure. Here, the CCTO particles are micro-sized CCTO particles.

composites with a higher CCTO content ( $>20$ vol\%) (named as Regime-II for later on discussion), the dielectric loss at low frequency is clearly higher than that of polymer matrix and increases with decreasing frequency. To further study the composition dependence of the dielectric properties at low frequency, the dielectric properties at $1 \mathrm{kHz}$ of these composites are plotted against its CCTO content as shown in Fig. 2. Although the dielectric constant of the composites increases with increasing CCTO content, the dielectric loss in both Regime-I and Regime-II remains almost a constant. Comparing composites in Regime-I and Regime-II, it is found that the composites in Regime-I exhibit an even lower dielectric loss. For all composites reported here, the experimental results indicate that the CCTO-P(VDF-CTFE) composites have a lower dielectric loss than other high dielectric constant composites. For example, the dielectric loss of CCTO-P(VDF-CTFE) composites reported here is mostly less than $15 \%$, while the CCTO-P(VDF-TrFE) composites exhibit a dielectric loss mostly higher than $30 \%$ at room temperature [20]. 


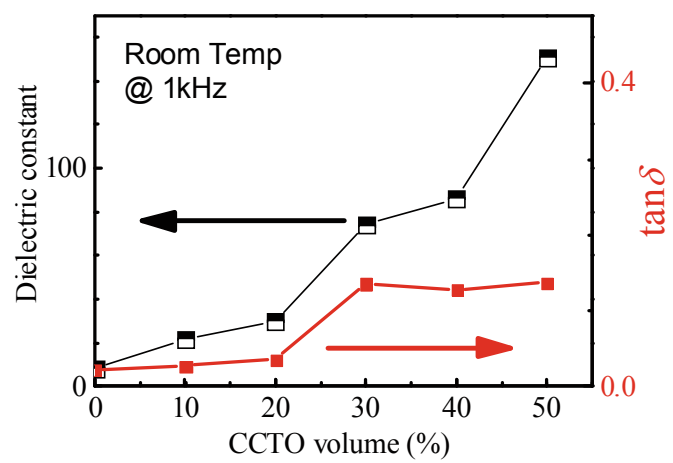

Fig. 2 Dielectric constant and dielectric loss at 1 $\mathrm{kHz}$ versus volume content of micro-sized CCTO for the composites at room temperature.

The temperature dependences of the dielectric constant are summarized in Fig. 3 and Fig. 4 for the composites studied here. In Fig. 3, the dielectric constant at $10 \mathrm{kHz}$ is plotted against temperature for all composites. As mentioned above, the dielectric property of $\mathrm{P}(\mathrm{VDF}-\mathrm{TrFE})$-based composites exhibits a strong temperature dependence due to the ferroelectricto-paraelectric phase transition. For example, for CCTO-P(VDF-TrFE) composites, the dielectric constant at the ferroelectric-to-paraelectric phase transition temperature of $\mathrm{P}(\mathrm{VDF}-\mathrm{TrFE})$ is about 2 to 3 times higher than that at room temperature $[20,21]$. Comparing to $\mathrm{P}(\mathrm{VDF}-\mathrm{TrFE})$-based composites, the composites reported here show an almost temperature independent dielectric constant, as shown in Fig. 3. To further study the temperature dependence of the dielectric properties, a temperature coefficient (Temp-Coef) is defined as

$$
\text { Temp-Coef }=\frac{\operatorname{Max}(\varepsilon)-\operatorname{Min}(\varepsilon)}{\operatorname{Max}(\varepsilon)+\operatorname{Min}(\varepsilon)}
$$

where $\operatorname{Max}(\varepsilon)$ and $\operatorname{Min}(\varepsilon)$ are the maximum and minimum values of the dielectric constant at a constant frequency over the temperature range from $25{ }^{\circ} \mathrm{C}$ to $125^{\circ} \mathrm{C}$. The Temp-Coef defined in Eq. (1) represents the maximum derivation of the dielectric constant from its median value. The value of Temp-Coef at some frequencies for different composites is summarized in Fig. 4. Comparing with other composites with high dielectric constant or composites using polar materials $[7,20,21]$, the composites studied here clearly exhibit a miniscale temperature dependence of the dielectric constant. The composites in Regime-I exhibit a smaller Tem-Coef than composites in Regime-II. More importantly, for the composites in Regime-I, the Temp-Coef decreases with increasing CCTO content.

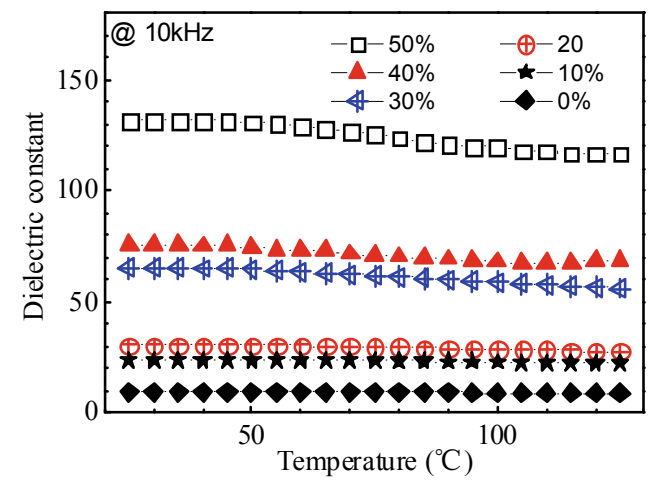

Fig. 3 Temperature dependence of dielectric constant at $10 \mathrm{kHz}$ for the composites with different contents of micro-sized CCTO particles.

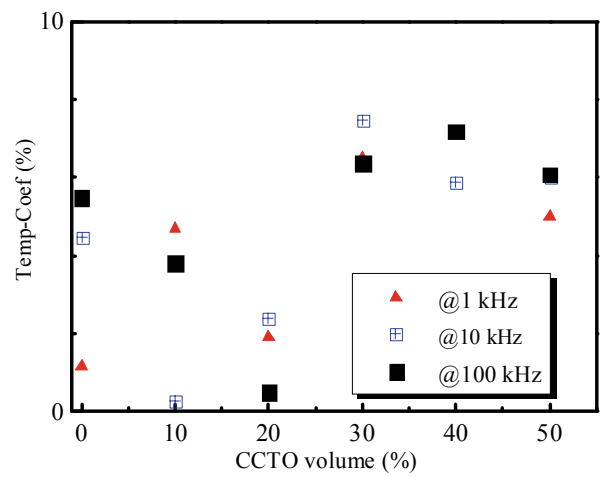

Fig. 4 The temperature coefficient (Temp-Coef) defined by Eq. (1) is plotted against the CCTO content for the composites.

That is, the composites have a smaller Temp-Coef than the pure polymer matrix.

The dielectric loss obtained in a dielectric may originate from electric conductivity and the dielectric relaxations, which includes interface relaxation for a composite. The dielectric loss originating from electrical conductivity is unacceptable for many of the dielectric applications, such as energy storage. It is well known that the temperature dependence of the dielectric loss reflects the nature of the dielectric loss observed in a dielectric. For dielectrics, the temperature dependence of the electrical conductivity can be written as

$$
\sigma=\sigma_{0} \cdot \exp \left(-\frac{T_{0}}{T}\right)
$$

where $\sigma$ and $\sigma_{0}$ are the conductivity at temperature $T$ and the conductivity at very high temperature, $T_{0}$ is the equivalent temperature of the activation energy of the conduction process in the dielectric. Therefore, the dielectric loss originating from the electrical conductivity would increase with increasing 


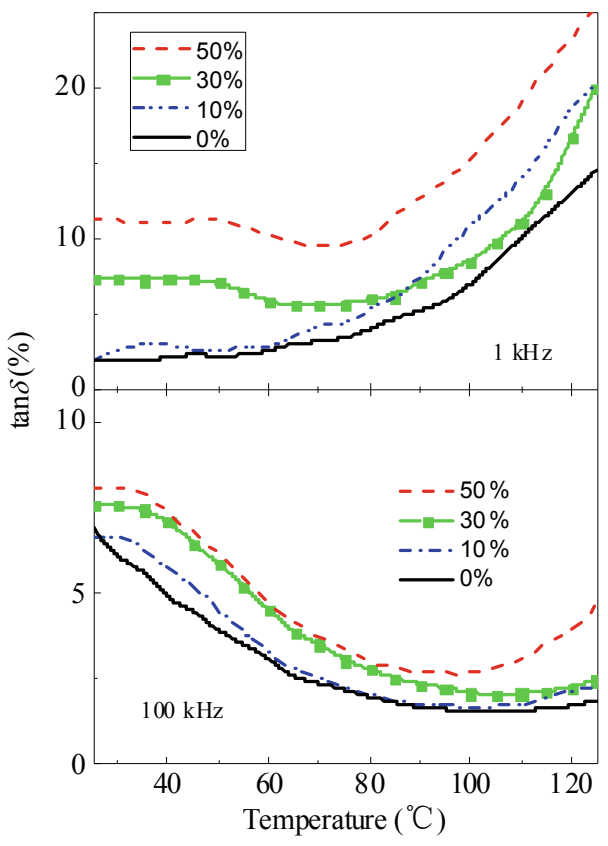

Fig. 5 Dielectric loss at $1 \mathrm{kHz}$ and $100 \mathrm{kHz}$ versus temperature for the composites.

temperature. The dielectric loss and its temperature of the composites are examined. The results shown in Fig. 5 are the dielectric loss at $1 \mathrm{kHz}$ and $100 \mathrm{kHz}$ for the composites at different temperatures. At $100 \mathrm{kHz}$, the dielectric loss of the composites decreases with increasing temperature, which indicates that the dielectric loss observed in the composites are dominated by the dielectric loss originates from the relaxations. In other words, these composites have insignificant electrical conductivity. At $1 \mathrm{kHz}$, there is a clear increase in the dielectric loss with increasing temperature at temperature higher than $70{ }^{\circ} \mathrm{C}$ for all composites and also for pure polymer matrix. At temperature below $70{ }^{\circ} \mathrm{C}$, for the composites in Regime-I, the dielectric loss shows a similar temperature dependence, while for the composites in Regime-II, the dielectric loss decrease with temperature.

It was reported that the size of CCTO particles plays an important role on the dielectric behavior of CCTO-P(VDF-TrFE) composites [21]. To examine influence of particle size of CCTO on the dielectric property of the composites and to verify the conclusion that the CCTO-P(VDF-CTFE) composites have an almost temperature-independent dielectric constant, the composites using nano-sized CCTO particles were studied. The results shown in Fig. 6 are the dielectric constant and loss of the composites with $50 \mathrm{vol} \%$ nano-sized CCTO. In the figure, the dielectric constant at $10 \mathrm{kHz}$ and dielectric loss at $1 \mathrm{kHz}$ are plotted for the composite with $50 \mathrm{vol} \%$ of micro-sized CCTO particles for the comparison purpose. The results shown in Fig. 6 reveals following conclusions. First, it is found that the composites using nano-sized CCTO particles exhibit a significantly smaller dielectric loss. For example, the nano-composite at room temperature has a dielectric loss of $4 \%$ at $1 \mathrm{kHz}$, while the micro-composite has more than $10 \%$. Secondly, the nano-composites exhibit a smaller dielectric constant than the micro-composites. This is consistent with the results obtained in CCTO-P(VDF-TrFE) composites [21]. Thirdly, the nano-composites exhibit a smaller Temp-Coef than the micro-composites. For example, for the dielectric constants at $1,10,100 \mathrm{kHz}$, the Temp-Coef is about $5 \%, 6 \%, 6 \%$, respectively, for the composite with $50 \mathrm{vol} \%$ of micro-sized CCTO, while it is $4.0 \%, 0.5 \%, 1.5 \%$, respectively, for the composite with 50 vol\% of nano-sized CCTO.

It is interesting to compare the difference in the dielectric property between the composites made of nano-sized and micro-sized CCTO particles. The dielectric property of a 0-3 composite is determined by the dielectric property of the matrix, filler, and the interfacial layer between the matrix and filler and by the morphology of the composite. Since the composites were prepared at temperature no more than $150{ }^{\circ} \mathrm{C}$, it is not expected that the CCTO itself changes during the composite process. Therefore, the interfacial layer between the CCTO particle and P(VDF-CTFE) matrix is $\mathrm{P}(\mathrm{VDF}-\mathrm{CTFE})$ polymer chains with different conformation and morphology than the polymer chains in the polymer matrix. Both nano- and microcomposites studied here have a relatively good uniformity or similar morphology except the size of the CCTO filler. Therefore, there are two possibilities for the difference in the dielectric property between the nano- and micro-composites shown in Fig. 6: (1) the nano-sized CCTO particles have a smaller dielectric constant and a lower dielectric loss than the micro-sized ССТО particles; (2) the interfacial layer has a lower dielectric constant and a lower dielectric loss than the polymer matrix. It is noticed that in literatures the interfacial layer in polymer-based composites is usually used to explain the increase in the dielectric constant with increasing interfacial layer [1]. That is, a higher dielectric constant is assigned to the interfacial layer. However, the CCTO-based composites show that for the composites with the same 


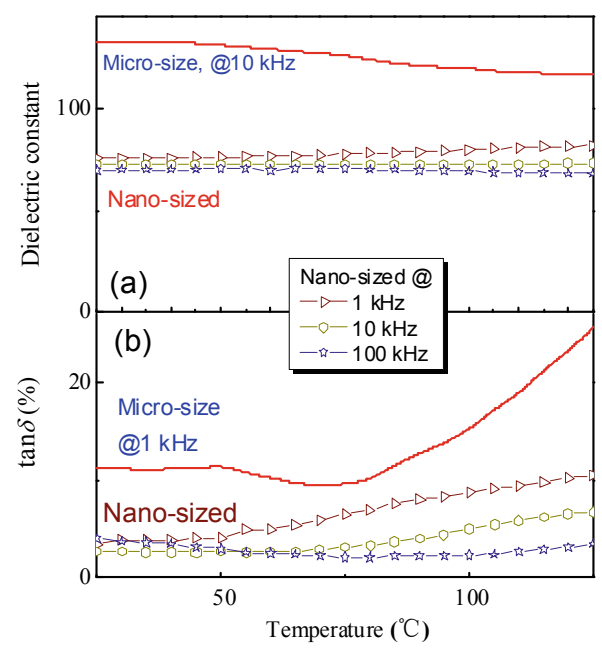

Fig. 6 Temperature dependence of dielectric constant (a) and loss (b) at different frequencies $(1,10,100$ $\mathrm{kHz}$ ) for CCTO-P(VDF-CTFE) composite with 50 vol\% of nano-sized CCTO. In the figure, the solid line without symbol is the results obtained in composites with $50 \mathrm{vol} \%$ of micro-sized CCTO particles.

polymer matrix and the same concentration, the composites using nano-sized CCTO particles exhibits a smaller dielectric constant and a lower dielectric loss than the composites using micro-sized CCTO particles. Therefore, these results indicate that the CCTO nano-sized particles have a smaller dielectric constant and a lower dielectric loss than the micro-sized CCTO particles. This may explain the experimental results that the dielectric property of the CСТO ceramics is sensitive to the microstructure.

In conclusion, 0-3 composites using micro-sized and nano-sized CCTO particles as filler are prepared using $\mathrm{P}$ (VDF-CTFE) copolymer as matrix. It is found that these composites exhibit a high dielectric constant with a low dielectric loss compared to the similar composites that use polar materials, such as ferroelectrics. More importantly, the dielectric constant obtained in CCTO-P(VDF-CTFE) composites is almost independent of temperature over temperature ranging from $25{ }^{\circ} \mathrm{C}$ to $125{ }^{\circ} \mathrm{C}$. Based on the dielectric property, the composition of the composites can be classified into two regimes: one is low CCTO content and the other is high CCTO content ( $>20$ vol\%). In each regime, the dielectric loss does not change much with the CCTO content. The composites in low CCTO content range exhibit a significant lower dielectric loss than the composites in high CCTO content range. It is also found that, for the composites in low CCTO content range, the temperature dependence of the dielectric constant becomes weaker as CCTO content increases. Comparing composites made using micro-sized CCTO particles, the composites made using nano-sized CCTO particle exhibit a significantly small dielectric loss and a weaker temperature dependence on the dielectric constant. It is concluded that the nano-sized CCTO particles have a smaller dielectric constant and a lower dielectric loss than the micro-sized CCTO particles.

\section{References}

[1] Zhang L, Cheng ZY. Development of polymer-based 0-3 composites with high dielectric constant. $J A d v$ Dielectrics 2011, 1: 389-406.

[2] Dang ZM, Yuan JK, Zha JW, et al. Fundamentals, processes and applications of high-permittivity polymer-matrix composites. Pro Mater Sci 2012, 57: 660-723.

[3] Sebastian MT, Jantunen H. Polymer-ceramic composites of $0-3$ connectivity for circuits in electronics: A Review. Int J Appl Ceram Technol 2010, 7: 415-434.

[4] Dang ZM, Wang HY, Peng B, et al. Effect of $\mathrm{BaTiO}_{3}$ size on dielectric property of $\mathrm{BaTiO}_{3} / \mathrm{PVDF}$ composites. J Electroceram 2008, 21: 381-384.

[5] Kuo DH, Chang CC, Su TY, et al. Dielectric properties of three ceramic/epoxy composites. Mater Chem Phys 2004, 85: 201-206.

[6] Lam KH, Chan HLW, Luo HS, et al. Dielectric properties of 65PMN-35PT/P(VDF-TrFE) 0-3 composites. Microelectron Eng 2003, 66: 792-797.

[7] Bai Y, Cheng ZY, Bharti V, et al. High dielectric constant ceramic powder polymer composites. Appl Phys Lett 2000, 76: 38043806.

[8] Dong LJ, Xiong CX, Quan HY, et al. Polyvinyl-butyral/lead zirconate titanates composites with high dielectric constant and low dielectric loss. Scr Mater 2006, 55: 835-837.

[9] Adikary SU, Chan HLW, Choy CL, et al. Characterisation of proton irradiated $\mathrm{Ba}_{0.65} \mathrm{Sr}_{0.35} \mathrm{TiO}_{3} / \mathrm{P}(\mathrm{VDF}-\mathrm{TrFE}) \quad$ ceramic-polymer composites. Compos Sci Technol 2002, 62: 2161-2167.

[10] Cheng ZY, Katiyar RS, Yao X, et al. Temperature dependence of dielectric constant of relaxor ferroelectrics. Phys Rev B 1998, 57: 8166.

[11] Cheng ZY, Zhang QM. Field actuated electroactive polymers. Mater Res Bull 2008, 33: 183-187. 
[12] Zhang QM, Bharti V, Zhao X. Giant electrostriction and relaxor ferroelectric behavior in electronirradiated poly (vinylidene fluoride-trifluoroethylene) copolymer. Science 1998, 280: 2101-2104.

[13] Li ZM, Li SQ, Cheng ZY. Crystalline structure and transition behavior of recrystallized-irradiated P(VDF-TrFE) 65/35 copolymer. J Appl Phys 2005, 97: 014102 .

[14] Li ZM, Arbatti MD, Cheng ZY. Recrystallization study of high-energy-electron irradiated $\mathrm{P}(\mathrm{VDF}-\mathrm{TrFE}) \quad 65 / 35$ copolymer. Macromolecules 2004, 37: 79-85.

[15] Cheng ZY, Zhang QM, Bateman FB. Dielectric relaxation behavior and its relation to microstructure in relaxor ferroelectric polymers - high-energy electron irradiated P(VDF-TrFE) copolymer. J Appl Phys 2002, 92: 6749-6755.

[16] Cheng ZY, Bharti V, Xu TB, et al. Electrostrictive poly (vinylidene fluoride-trifluoroethylene) copolymers. Sens Actuators A: Phys 2001, 90: 138-147.

[17] Subramanian MA, Li D, Duan N, et al. High dielectric constant in $\mathrm{ACu}(3) \mathrm{Ti}(4) \mathrm{O}(12)$ and $\mathrm{ACu}(3) \mathrm{Ti}(3) \mathrm{FeO}(12)$ phases. J Solid State Chem 2000, 151: 323-325.

[18] Homes CC, Vogt T, Shapiro SM, et al. Optical response of high-dielectric-constant perovskite-related oxide. Science 2001, 293: 673-676.

[19] Subramanian MA, Sleight AW. ACu(3)Ti(4)O(12) and $\mathrm{ACu}(3) \mathrm{Ru}(4) \mathrm{O}(12)$ perovskites: High dielectric constants and valence degeneracy. Solid State Sci 2002, 4: 347-351.

[20] Arbatti MD, Shan XB, Cheng ZY. Ceramic-polymer composites with high dielectric Constant. Adv Mater 2007, 19: 1369 .
[21] Zhang L, Shan XB, Wu PX, et al. Dielectric characteristics of $\mathrm{CaCu}_{3} \mathrm{Ti}_{4} \mathrm{O}_{12} / \mathrm{P}(\mathrm{VDF}-\mathrm{TrFE})$ nanocomposites. Appl Phys A 2012, 107: 597-602.

[22] Thomas P, Varughese KT, Dwarakanath K, et al. Dielectric properties of poly (vinylidene fluoride)/ $\mathrm{CaCu}_{3} \mathrm{Ti}_{4} \mathrm{O}_{12}$ composites. Compos Sci Technol 2010, 70: $539-545$

[23] Amaral F, Rubinger CPL, Henry F, et al. Dielectric properties of polystyrene-CCTO composite. $J$ Non-Cryst Sol 2008, 354: 5321-5322.

[24] Dang ZM, Zhou T, Yao SH, et al. Advanced calcium copper titanate/polyimide functional hybrid films with high dielectric permittivity. Adv Mater 2009, 21: 2077-2082.

[25] Li ZM. Novel electroactive poly (vinylidene fluoride)-based polymer systems and their applications. Ph.D. Dissertation. Auburn University, 2004.

[26] Li ZM, Wang YH, Cheng ZY. Electromechanical properties of poly (vinylidene-fluoridechlorotrifluoroethylene) copolymer. Appl Phys Lett 2006, 88: 062904.

[27] Chu BJ, Zhou X, Ren KL, et al. A dielectric polymer with high electric energy density and fast discharge speed. Science 2006, 313: 334-336.

[28] Li JJ. Nanocomposites based on ferroelectric polymers for electrical energy storage $\mathrm{Ph}$. D. Dissertation. Penn State University, 2009.

[29] Wang Q, Zhu L. Polymer nanocomposites for electrical energy storage. J Polym Sci 2011, B49: 1421-1429.

[30] Xia WM, Xu Z, Wen F, et al. Electrical energy density and dielectric properties of poly (vinylidene fluoride-chlorotrifluoroethylene)/BaSrTiO3 nanocomposites. Ceram Int 2012, 38: 1071-1075. 